\section{MS39-P5 Neutron macromolecular crystallography at the FRM II - The neutron single crystal diffractometer BIODIFF}

\author{
Andreas Ostermann ${ }^{1}$, Tobias E. Schrader ${ }^{2}$, Michael \\ Monkenbusch ${ }^{2,3}$, Bernhard Laatsch ${ }^{4}$, Philipp Jüttner ${ }^{5}$, Winfried \\ Petry $^{1}$, Dieter Richter ${ }^{2,3}$
}

1. Heinz Maier-Leibnitz Zentrum (MLZ), Technische Universität München, Lichtenbergstr.1, 85748 Garching, Germany

2. Jülich Centre for Neutron Science JCNS, Forschungszentrum Jülich GmbH, Outstation at MLZ, Lichtenbergstr.1, 85747 Garching, Germany

3. Institute for Complex Systems ICS, Forschungszentrum Jülich GmbH, 52425 Jülich, Germany

4. Forschungszentrum Jülich $\mathrm{GmbH}$, Engineering and Technology (ZEA-1), 52425 Jülich, Germany

5. Forschungs-Neutronenquelle Heinz Maier-Leibnitz (FRM II), Technische Universität München, Lichtenbergstrasse 1, 85748 Garching

email: Andreas.Ostermann@frm2.tum.de

The research reactor Heinz Maier-Leibnitz (FRM II) is a modern high flux neutron source which feeds at the present 27 state of the art instruments. The newly build neutron single crystal diffractometer BIODIFF is especially designed to collect data from crystals with large unit cells. The main field of application is the structure analysis of proteins, especially the determination of hydrogen atom positions. BIODIFF is a joint project of the Forschungszentrum Jülich (FZJ/JCNS) and the Forschungs-Neutronenquelle Heinz Maier-Leibnitz (FRM II). Typical scientific questions addressed are the determination of protonation states of amino acid side chains and the characterization of the hydrogen bonding network between the protein and an inhibitor or substrate. BIODIFF is designed as a monochromatic instrument. By using a highly orientated pyrolytic graphite monochromator (PG002) the diffractometer is able to operate in the wavelength range of $2.4 \AA$ to about $5.6 \AA$. Contaminations of higher order wavelengths are removed by a neutron velocity selector. To cover a large solid angle and thus to minimize the data collection time the main detector of BIODIFF consists of a neutron imaging plate system in a cylindrical geometry. A $\mathrm{Li} / \mathrm{ZnS}$ scintillator CCD camera is available for additional detection abilities. The main advantage of BIODIFF is the possibility to adapt the wavelength to the size of the unit cell of the sample crystal while operating with a clean monochromatic beam that keeps the background level low. BIODFF is equipped with a standard Oxford Cryosystem "Cryostream 700+" which allows measurements in the temperature regime from $90 \mathrm{~K}$ up to $500 \mathrm{~K}$.

Keywords: Neutron protein crystallography, hydrogen atom positions, protonation states, hydrogen bonds, ligand binding, hydration structure

\section{MS39-P6 Upgrading home-lab X-ray diffractometers with Incoatec's unique microfocus source $\mathrm{I} \mu \mathrm{S}$}

André Beerlink ${ }^{1}$, Jürgen Graf ${ }^{1}$, Jörg Wiesmann ${ }^{1}$, Carsten Michaelsen $^{1}$

1. Incoatec GmbH, Max-Planck-Str. 2, 21502 Geesthacht, Germany

email: beerlink@incoatec.de

Modern microfocus X-ray sources define the state-of-the-art for a broad spectrum of applications in home laboratories, such as protein and small molecule crystallography, and small-angle scattering. These sources are combined with multilayer Montel optics to image the source spot onto the sample. These optics provide a parallel or focused monochromatic X-ray beam, magnified to a suitable size.

Low power sealed microfocus sources, such as Incoatec's I $\mu$ S represent an attractive alternative to rotating anodes, with a significant reduction in cost and maintenance. Power loads of a few $\mathrm{kW} / \mathrm{mm}^{2}$ in anode spot sizes below 50 micrometer diameter deliver a compact and very brilliant beam. For example, the $\mathrm{I} \mu \mathrm{S}^{\text {HighBrilliance }}$ delivers more than $2 * 10^{10}$ photons $/ \mathrm{s} / \mathrm{mm}^{2}$ in a focal spot size of below 100 micrometer diameter at the sample position. It is available for $\mathrm{Cu}, \mathrm{Mo}, \mathrm{Ag}, \mathrm{Cr}$ and $\mathrm{Co}$ anodes. Since the launch in 2006 nearly $600 \mathrm{I} \mu \mathrm{S}$ systems are now in operation worldwide for a large variety of applications in biology, chemistry, physics and material science.

Are you tired of getting spare parts for an ancient rotating anode or is your detector performance only limited by your x-ray source that lacks intensity? We will demonstrate how to bring former high end diffractometers back to a superb performance for cutting edge science after an upgrade with a high performance $\mathrm{I} \mu \mathrm{S}$ source. Incoatec ensures full software and safety integration, and an installation hand in hand with your local service responsible, providing a constant service support by your partners on site.

In addition to all Bruker and Nonius systems, Incoatec also offers integrations into a wide range of instruments, e.g. Rigaku, marXperts, STOE, also together with Dectris or Huber components and we also provide customized special engineering solutions.

Keywords: microfocus, X-ray source, Incoatec, Scatex, brilliance, crystallography, protein, chemical 